\title{
Performance Evaluation of the Stat Profile pHOx Ultra Blood Gas Analyzer
}

Hye-Young Lee, Sunyoung Ahn, HyunKi Kim, Woochang Lee, Sail Chun, and Won-Ki Min

Department of Laboratory Medicine, Asan Medical Center, University of Ulsan College of Medicine, Seoul, Korea

\author{
Corresponding author: \\ Won-Ki Min \\ Department of Laboratory \\ Medicine, Asan Medical Center, \\ University of Ulsan College of \\ Medicine, 88 Olympic-ro 43-gil, \\ Songpa-gu, Seoul 05505, Korea \\ Tel: $+82-2-3010-4503$ \\ Fax: +82-2-478-0884 \\ E-mail: wonkmin@gmail.com
}

pISSN: 2384-2458

eISSN: 2288-7261

\begin{abstract}
The objective of this study was to evaluate the analytical performance of the Stat Profile pHOx Ultra Blood Gas Analyzer (Nova Biomedical, USA), a new blood gas/chemistry analyser, including its precision and linearity, comparison studies, and the carry-over effect of commercial reagents and patient specimens. We assessed all the results on the basis of the Clinical and Laboratory Standards Institute guidelines. The following parameters were assessed: $\mathrm{pH}$, partial pressure of carbon dioxide, partial pressure of oxygen, ionized calcium, ionized magnesium (iMg), and lactate concentration The total imprecision had a coefficient of variation of $0.0 \%-1.8 \%$, and the linear measurement ranges for each parameter were all acceptable. In comparison with the Nova Critical Care Xpress Analyzer (Nova Biomedical, USA), the results indicated a good agreement, except for iMg. All carry-over ranges were between $-0.5 \%$ and $-1.4 \%$. The Stat Profile pHOx Ultra Blood Gas Analyzer showed good analytical performance in terms of precision, linearity, comparison studies, and carry-over effect. The Stat Profile pHOx Ultra Blood Gas Analyzer can provide reliable measurements across a clinically relevant range and has potential use in laboratory tests.
\end{abstract}

(J Lab Med Qual Assur 2019;41:47-49)

Key Words: Stat Profile pHOx Ultra Blood Gas Analyzer, Performance, Evaluation

Received October 23, 2018, Revision received February 28, 2019, Accepted March 4, 2019
혈액가스, 전해질 및 대사산물 측정 분석기기인 Stat Profile pHOx Ultra Blood Gas Analyzer (Nova Biomedical, Waltham, MA, USA)의 분석성능을 평가하였다. 평가는 $\mathrm{pH}$, partial pressure of carbon dioxide $\left(\mathrm{pCO}_{2}\right)$, partial pressure of oxygen $\left(\mathrm{pO}_{2}\right)$, ionized calcium (iCa), ionized magnesium (iMg), lactate로 총 6 개 검사항목에 대하여 실시하였다. 정 밀도평가에서 $\mathrm{pH}, \mathrm{pCO}_{2}, \mathrm{pO}_{2}$ 는 저농도, 중간농도, 고농도 로 구성된 Stat Profile pHOx Ultra/CCX blood gas controls (Nova Biomedical) level 1-3을, iCa, iMg, lactate은 저농도, 고농도로 구성된 Stat Profile pHOx Ultra/CCX chemistry controls (Nova Biomedical) level 1-2를 이용하였다. 총 정밀 도와 검사일 간 정밀도는 Clinical and Laboratory Standards Institute (CLSI) EP05-A3에 따라 각각의 정도관리물질을 20 일 동안 매일 2회, 매회 2회 반복 측정하여 구하였다[1,2]. 직선성 평가는 상품화된 Calibration Verification Controls
(RNA Medical, Devens, CA, USA)을 이용하여 5가지 농 도로 평가하였는데, 이들을 각각 4회씩 반복 측정하여 CLSI $\mathrm{EP} 06-\mathrm{A}$ 에 따라 평가하였다 $[1,3]$. 상관성 평가는 충분한 양 의 환자 검체 40개를 이용하여 CLSI EP09-A3에 따라 Nova Critical Care Xpress (CCX) Analyzer (Nova Biomedical) 와 평가기기에서 차례로 각각 2회씩 반복 측정한 후 그 결과 를 분석하였다[4,5]. Nova Biomedical사의 상품화된 정도 관리물질 high/low level로 검체 간 상호오염 백분율(carryover percentage)을 계산하였다 [1,6]. 각 항목별 정밀도 검사 결과에서 총 정밀도는 $0.0 \%$ 에서 $1.8 \%$ 이었으며 검사일 간 정 밀도는 $0.0 \%$ 에서 $1.3 \%$ 였다(Table 1 ). 직선성 평가결과를 위 한 회귀분석결과 모든 검사항목에서 결정계수 $\left(R^{2}\right)$ 가 0.9961.000으로 나타났다(Fig. 1). Nova CCX Analyzer (Nova Biomedical $)$ 와 비교평가에서 $\mathrm{iMg}(r=0.974)$ 를 제외한 상관 계수 $(r)$ 값이 0.976-0.999로 상관성을 만족하였고, 6개 항목 
Table 1. Precision profile of the Stat Profile pHOx Ultra Blood Gas Analyzer

\begin{tabular}{|c|c|c|c|c|c|c|}
\hline \multirow{2}{*}{ Analyte } & \multirow[b]{2}{*}{ Level } & \multirow{2}{*}{$\begin{array}{c}\text { Mean } \\
(\mathrm{mg} / \\
\mathrm{dL})\end{array}$} & \multicolumn{4}{|c|}{ CV (\%) } \\
\hline & & & $\begin{array}{l}\text { Wi- } \\
\text { thin } \\
\text { run }\end{array}$ & $\begin{array}{c}\text { Bet- } \\
\text { ween } \\
\text { run }\end{array}$ & $\begin{array}{l}\text { Bet- } \\
\text { ween } \\
\text { day }\end{array}$ & Total \\
\hline \multirow[t]{3}{*}{$\mathrm{pH}$} & Low & 7.2 & 0.0 & 0.0 & 0.0 & 0.0 \\
\hline & Middle & 7.4 & 0.0 & 0.0 & 0.0 & 0.0 \\
\hline & High & 7.6 & 0.0 & 0.0 & 0.0 & 0.0 \\
\hline \multirow[t]{3}{*}{$\mathrm{pCO}_{2}(\mathrm{~mm} \mathrm{Hg})$} & Low & 23.2 & 0.7 & 0.9 & 0.7 & 0.5 \\
\hline & Middle & 41.7 & 0.6 & 0.5 & 0.3 & 0.8 \\
\hline & High & 54.9 & 0.7 & 0.3 & 0.3 & 1.3 \\
\hline \multirow[t]{3}{*}{$\mathrm{pO}_{2}(\mathrm{~mm} \mathrm{Hg})$} & Low & 56.5 & 0.8 & 0.0 & 0.4 & 0.6 \\
\hline & Middle & 117.7 & 0.2 & 0.2 & 0.2 & 0.4 \\
\hline & High & 141.2 & 0.2 & 0.3 & 0.5 & 0.9 \\
\hline \multirow[t]{2}{*}{$\mathrm{iCa}(\mathrm{mg} / \mathrm{dL})$} & Low & 4.3 & 0.4 & 0.0 & 0.1 & 0.4 \\
\hline & High & 6.4 & 0.3 & 0.2 & 0.0 & 0.3 \\
\hline \multirow[t]{2}{*}{$\mathrm{iMg}(\mathrm{mg} / \mathrm{dL})$} & Low & 1.6 & 1.6 & 0.0 & 0.8 & 1.8 \\
\hline & High & 2.9 & 1.2 & 0.0 & 1.3 & 1.8 \\
\hline \multirow[t]{2}{*}{ Lactate $(\mathrm{mmol} / \mathrm{L})$} & Low & 2.0 & 0.6 & 0.0 & 0.0 & 0.6 \\
\hline & High & 7.0 & 0.6 & 0.2 & 1.3 & 1.5 \\
\hline
\end{tabular}

The instrument and quality control materials used in this study were from Stat Profile pHOx Ultra Blood Gas Analyzer (Nova Biomedical, Waltham, MA, USA) and Calibration Verification Controls (RNA Medical, Devens, CA, USA), respectively.

Abbreviations: $\mathrm{CV}$, coefficient of variation; $\mathrm{pCO}_{2}$, partial pressure of carbon dioxide; $\mathrm{pO}_{2}$, partial pressure of oxygen; $\mathrm{iCa}$, ionized calcium; iMg, ionized magnesium.
의 회귀곡선의 기울기는 0.962-1.091, $y$ 절편은 $-0.630-0.630$ 이었다(Fig. 2). 모든 상호오염 백분율의 범위는 $-0.5 \%-$ $1.4 \%$ 이었다(Table 2). Stat Profile pHOx Ultra Blood Gas Analyzer는 모든 검사항목에서 우수한 정밀도와 직선성을 보 여주었고, Nova CCX analyzer와 전반적으로 상관성도 높았 으며 상호오염 효과(carry-over effect)도 관찰되지 않았다. 결론적으로, Stat Profile pHOx Ultra Blood Gas Analyzer (Nova Biomedical)는 본 평가에서 우수한 분석성능을 보여주 어 임상검사실에서 유용하게 사용할 수 있을 것으로 생각된다.

Table 2. Carry-over effect on the measurement of the Stat Profile pHOx Ultra Blood Gas Analyzer with quality control material

\begin{tabular}{lc}
\hline \multicolumn{1}{c}{ Analyte } & Mean (\%) \\
\hline $\mathrm{pH}$ & -1.3 \\
$\mathrm{PCO}_{2}(\mathrm{~mm} \mathrm{Hg})$ & -0.5 \\
$\mathrm{PO}_{2}(\mathrm{~mm} \mathrm{Hg})$ & -1.4 \\
$\mathrm{iCa}(\mathrm{mg} / \mathrm{dL})$ & -1.1 \\
$\mathrm{iMg}(\mathrm{mg} / \mathrm{dL})$ & -0.5 \\
Lactate $(\mathrm{mmol} / \mathrm{L})$ & -1.0 \\
\hline
\end{tabular}

Abbreviations: $\mathrm{pCO}_{2}$, partial pressure of carbon dioxide; $\mathrm{pO}_{2}$, partial pressure of oxygen; iCa, ionized calcium; iMg, ionized magnesium.
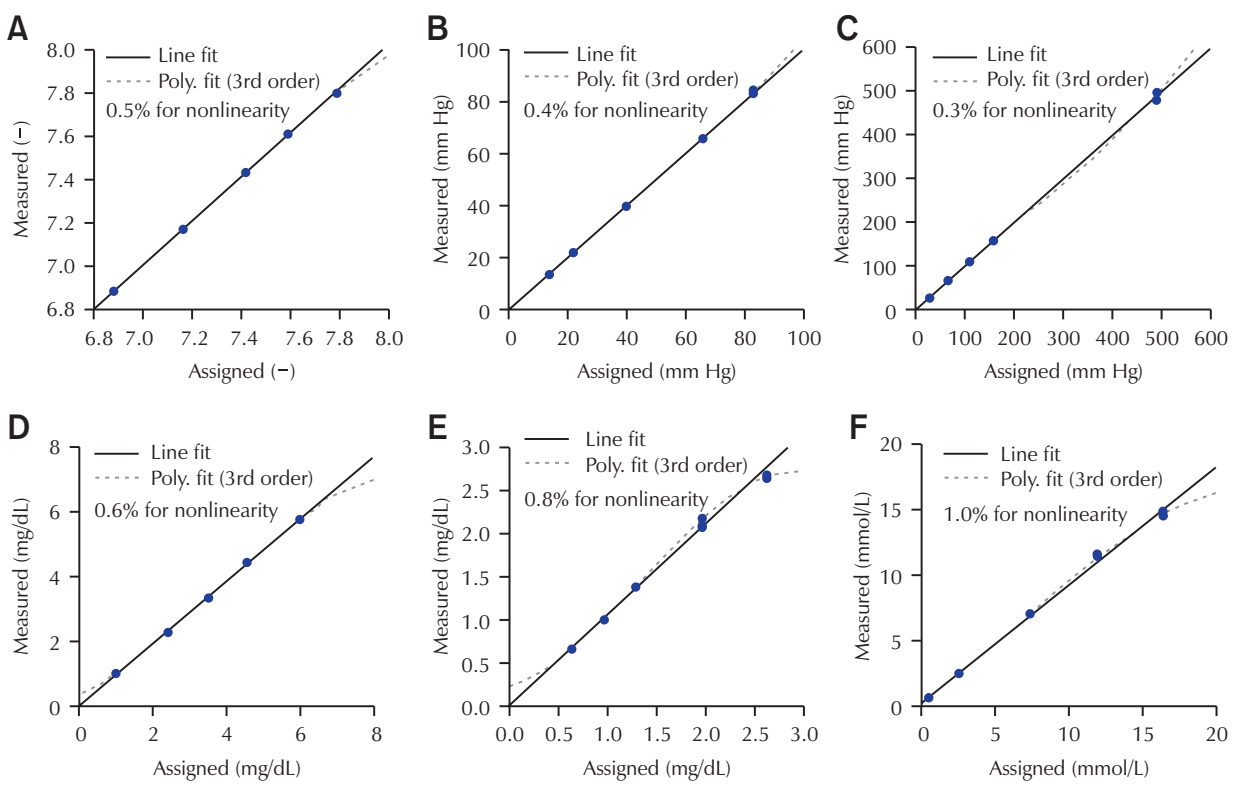

Fig. 1. Linearity profile on the Stat Profile pHOx Ultra Blood Gas Analyzer. (A) $\mathrm{pH},(\mathrm{B}) \mathrm{pCO}_{2}$, (C) $\mathrm{pO}_{2}$, (D) $\mathrm{iCa},(\mathrm{E}) \mathrm{iMg},(\mathrm{F})$ lactate. $\mathrm{Ab}-$ breviations: $\mathrm{pCO}_{2}$, partial pressure of carbon dioxide; $\mathrm{pO}_{2}$, partial pressure of oxygen; $\mathrm{iCa}$, ionized calcium; $\mathrm{iMg}$, ionized magnesium. 

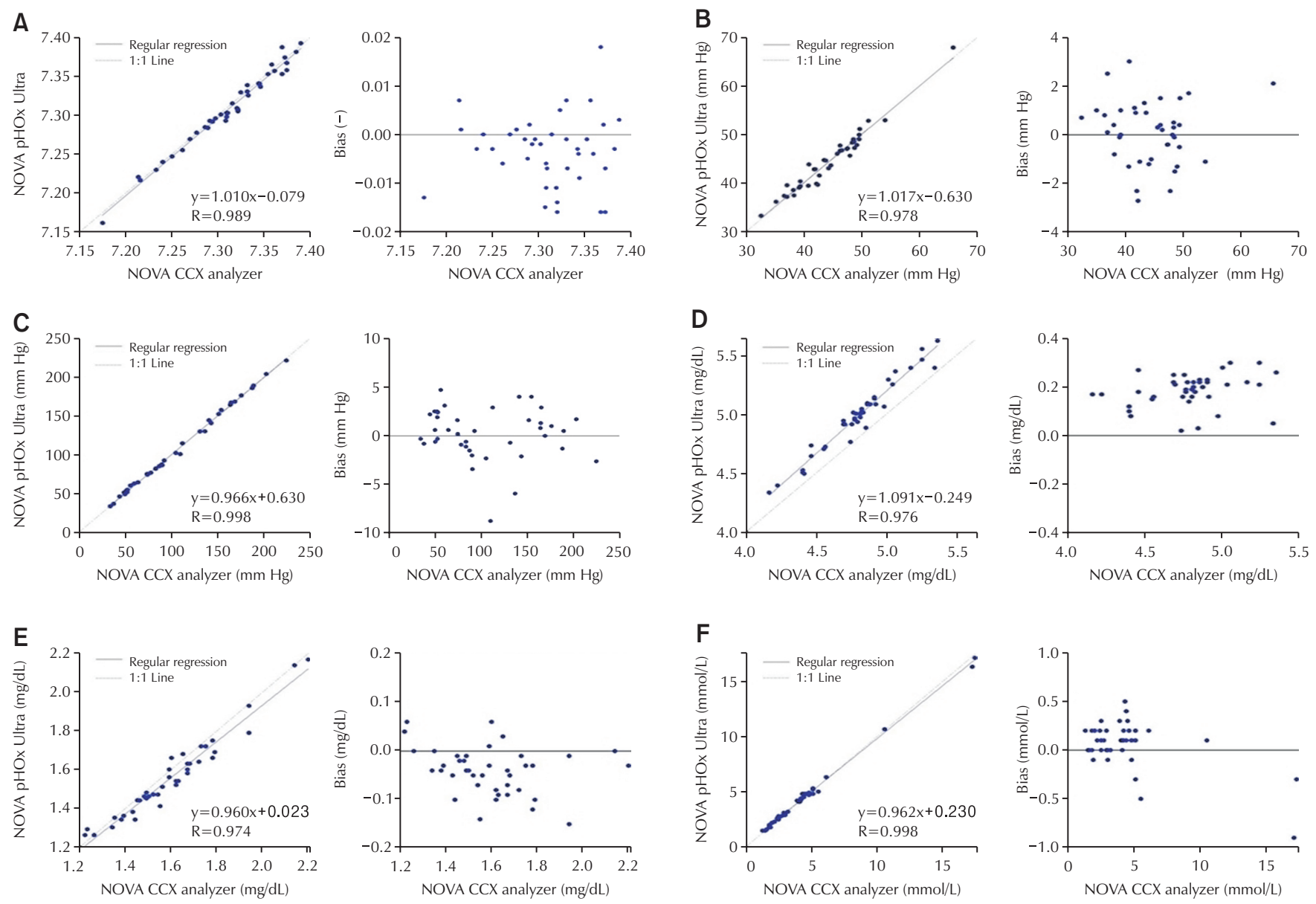

Fig. 2. Comparison of the Nova Critical Care Xpress Anlayzer and Nova pHOx Ultra Blood Gas Anlayzer. Scatter plot and bias plot are shown for each analyte. In scatter plots, solid lines indicate regression lines and dotted lines indicate identity lines. (A) $\mathrm{pH},(\mathrm{B}) \mathrm{pCO}_{2},(\mathrm{C}) \mathrm{pO}_{2},(\mathrm{D}) \mathrm{iCa},(\mathrm{E}) \mathrm{iMg},(\mathrm{F})$ lactate. Abbreviations: $\mathrm{pCO}_{2}$, partial pressure of carbon dioxide; $\mathrm{pO}_{2}$, partial pressure of oxygen; $\mathrm{iCa}$, ionized calcium; iMg, ionized magnesium.

\section{REFERENCES}

1. Nova Biomedical. Nova pHOx Ultra Blood Gas Analyzer instructions for use manual (ref 49795). Waltham (MA): Nova Biomedical, 2018.

2. Clinical and Laboratory Standards Institute. Evaluation of precision performance of quantitative measurement methods: approved guideline. 3rd ed. Wayne (PA): Clinical and Laboratory Standards Institute, 2014.

3. Clinical and Laboratory Standards Institute. Evaluation of the linearity of quantitative measurement procedures: a statistical approach: approved guideline. Wayne (PA):
Clinical and Laboratory Standards Institute, 2003.

4. Clinical and Laboratory Standards Institute. Measurement procedure comparison and bias estimation using patient samples: approved guideline. 3rd ed. Wayne (PA): Clinical and Laboratory Standards Institute, 2013.

5. Nova Biomedical. Nova Critical Care Xpress (CCX) Analyzer instructions for use manual (ref 38343). Waltham (MA): Nova Biomedical, 2017.

6. Pesce MA. Laboratory automation. In: Kaplan LA, Pesce AJ, editors. Clinical chemistry: theory, analysis, correlation. 5th ed. St. Louis (MO): Mosby, 2010:370. 